\title{
Anatomical mechanism of spontaneous recovery in regions caudal to thoracic spinal cord injury lesions in rats
}

\author{
Lu-sheng Li ${ }^{1}$, Hao Yu ${ }^{1}$, Raynald Raynald ${ }^{2}$, Xiao-dong Wang ${ }^{2}$, Guang-hui Dai ${ }^{2}$, Hong-bin Cheng ${ }^{2}$, Xue-bin \\ Liu ${ }^{2}$, Yi-hua An ${ }^{\text {Corresp. } 1,2}$ \\ 1 Department of Neurosurgery, Beijing Sanbo Brain Hospital, Capital Medical University, Beijing, China \\ 2 Department of Functional Neurosurgery and Cytotherapy, General Hospital of Chinese People's Armed Police Forces, Beijing, China \\ Corresponding Author: Yi-hua An \\ Email address: anyihua_wj@sina.com
}

Background: The nerve fibre circuits around a lesion play a major role in the spontaneous recovery process after spinal cord hemisection in rats. The aim of the present study is: in the re-control process, do all spinal cord nerves below the lesion site participate, or do the spinal cord nerves of only one vertebral segment have a role in repair? Methods: First we made a T7 spinal cord hemisection in 50 rats. Eight weeks later, they were divided into 3 groups based on distinct second operations at T7: ipsilateral hemisection operation, contralateral hemisection, or transection. We then tested recovery of hindlimbs for another 8 weeks. The first step was to confirm the lesion had role or not in the spontaneous recovery process. Secondly, we performed T7 spinal cord hemisections in 125 rats. Eight weeks later, we performed a second single hemisection on the ipsilateral side at T8-T12 and then tested hindlimb recovery for another 6 weeks. Results: In the first part, the Basso, Beattie, Bresnahan (BBB) scores and the electrophysiology tests of both hindlimbs weren't significantly different after the second hemisection of the ipsilateral side. In the second part, the closer the second hemisection was to T12, the more substantial the resulting impairment in BBB score tests and prolonged latency periods. Conclusions: The nerve regeneration from the lesion area after hemisection has no effect on spontaneous recovery of the spinal cord. Repair is carried out by all vertebrae caudal and ipsilateral to the lesion, with T12 being most important. 


\section{Anatomical mechanism of spontaneous recovery in regions caudal to}

\section{2 thoracic spinal cord injury lesions in rats}

3

\section{Authors:}

5 Lu-sheng Li ${ }^{1}$, Hao Yu ${ }^{1}$, Raynald Raynald ${ }^{2}$, Xiao-dong Wang ${ }^{2}$, Guang-hui Dai ${ }^{2}$, Hong-bin

6 Cheng ${ }^{2}$, Xue-bin Liu ${ }^{2}$, Yi-hua An ${ }^{1,2, *}$

7

\section{Affiliations:}

91 Department of Neurosurgery, Beijing Sanbo Brain Hospital, Capital Medical University, 10 Beijing 100093, China

$112^{2}$ Department of Functional Neurosurgery and Cytotherapy, General Hospital of Chinese People's

12 Armed Police Forces, Beijing 100039, China

13 * Corresponding author; Department of Stem Cell Transplantation, The General Hospital of 14 Chinese People's Armed Police Forces, Beijing 100039, China.

15 E-mail addresses: anyihua_wj@sina.com (Yi-hua An).

Fax number: +0086-10-57976848 


\section{Abstract}

Background: The nerve fibre circuits around a lesion play a major role in the spontaneous recovery process after spinal cord hemisection in rats. The aim of the present study is: in the recontrol process, do all spinal cord nerves below the lesion site participate, or do the spinal cord nerves of only one vertebral segment have a role in repair?

Methods: First we made a T7 spinal cord hemisection in 50 rats. Eight weeks later, they were divided into 3 groups based on distinct second operations at T7: ipsilateral hemisection operation, contralateral hemisection, or transection. We then tested recovery of hindlimbs for another 8 weeks. The first step was to confirm the lesion had role or not in the spontaneous recovery process. Secondly, we performed T7 spinal cord hemisections in 125 rats. Eight weeks later, we performed a second single hemisection on the ipsilateral side at T8-T12 and then tested hindlimb recovery for another 6 weeks.

Results: In the first part, the Basso, Beattie, Bresnahan (BBB) scores and the electrophysiology tests of both hindlimbs weren't significantly different after the second hemisection of the ipsilateral side. In the second part, the closer the second hemisection was to T12, the more substantial the resulting impairment in BBB score tests and prolonged latency periods.

Conclusions: The nerve regeneration from the lesion area after hemisection has no effect on spontaneous recovery of the spinal cord. Repair is carried out by all vertebrae caudal and ipsilateral to the lesion, with T12 being most important.

Keywords: spinal cord injury, hemisection, transection, recovery, electrophysiology, nerve 
42

43

44

\section{Introduction}

Brain is plastic and mammals are capable of spontaneous recovery after spinal cord injury. The mechanisms underlying this process are not yet clear and are disputed. After brain or spinal cord injury, many researchers have found that transplantation of multifunctional three-dimensional scaffolds and stem cells treatment with neurotrophic factors, administration of small molecules, or genetic modifications in the lesion area promote neuronal regeneration in the lesion and improve motor function recovery (Estrada et al. 2014; Fan et al. 2010; Jee et al. 2012; McCall et al. 2012; Shi et al. 2015; Tan et al. 2016; Wright et al. 2011). In addition, several groups have shown that, after hemisection of the thoracic spinal cord, both hindlimbs show significant improvement 3-5 weeks later. Moreover, if a second hemisection on the side contralateral to the lesion was performed, rats showed complete paralysis of both hind limbs with no signs of recovery of locomotor function over 4 weeks. For example, Courtine et al. first produced a leftside hemisection model at thoracic segment 12 (T12 refers to the spinal cord level). Then, 10 weeks later, they performed a second hemisection on the contralateral side (T7). The results showed that the rats initially lost all movement on the T7 side and some of the movement on the T12 side. However, when the rats were subjected to the T7 and T12 hemisection at the same time, both hindlimbs instantly lost all movement (Courtine et al. 2008). This observation demonstrates that nerve fibers around the lesion participate in repair. These fibers must originate rostral to the lesion on the ipsilateral side, then cross the midline to the contralateral side, travel down the spinal cord, and re-cross the midline caudal to the lesion (Ballermann \& Fouad 2006; Courtine et al. 2008; Etlin et al. 2010; Reed et al. 2008). These two different repair mechanisms are in conflict with each other and further research is needed to confirm and explain the repair process. If the mechanisms of repair in both contexts were resolved it would inform novel 
65 66 injury.

67

treatments to promote recovery and further improve limb function in patients with spinal cord

Among the questions that remain to be answered is whether the nerve fiber circuits that control the ipsilateral hindlimb after injury are comprised of spinal cord nerves in a single vertebra or spinal cord nerves in multiple vertebrae contribute to these nerve fiber circuits and participate in the recovery process. Accordingly, we designed a study to resolve these two possibilities. We first carried out a hemisection of thoracic vertebrae 7 (T7) on the left side of spinal cord, then 8 weeks later performed a second hemisection of thoracic vertebrae 8-12 (T8T12) on the ipsilateral side. We then compared the extent of recovery of hindlimb function acrosss each hemisection group. In this study, abbreviations such as T7 indicate the vertebral segments.

\section{Materials and Methods}

\subsection{Animals}

In this study, we used adult Sprague-Dawley rats (200-220 g). All rats were allowed to acclimate to the new environment for 7 days before the start of any experimental procedures. The rats were housed on a $12 \mathrm{~h}$ light-dark cycle with food and water provided ad libitum. All rats were deeply anesthetized before any surgical procedures were performed $(10 \%$ chloral hydrate, $3.5 \mathrm{ml} / \mathrm{kg}$ ). After surgery, antibiotic (Penicillin, 128000UI/kg) and $10 \mathrm{ml}$ of sterile saline were administered subcutaneously each day during the first week. After each operation, the rat was placed in a separate cage for 7 days before it was housed with other rats. The rats in the study were obtained from the Vital River Company. Ethical approval was obtained from the Beijing Neurosurgical Institute Laboratory Animals Ethics Committee in China. 
88

89

90

91

92

93

94

95

96

97

In the first part of study, there were 50 rats in total and 10 rats died throughout the study, the mortality was $20 \%$. In the second part of study, there were 125 rats in total and 16 rats died throughout the study, the mortality was $12.8 \%$.

\subsection{Groups}

In the first part of the study, we investigated if regenerated axons coursed ipsilaterally or contralaterally. All rats underwent two operations on $\mathrm{T} 7$ spinal cord and were divided into 5 groups (Fig. 1A-E). The first group was a sham operation group $(\mathrm{N}=10)$ in which both operations were sham. Groups 2-5 all first received a hemisection of the left side of spinal cord at T7, but had different second operations. The second group was the control group $(\mathrm{N}=6)$, in which the second operation was sham, consisted a midline cut in the spinal cord at T7. The third group was the ipsilateral experimental group $(\mathrm{N}=8)$, in which a second hemisection was conducted on the ipsilateral side. The fourth group was the contralateral group $(\mathrm{N}=8)$, in which a second hemisection was made on the contralateral side. The fifth was the transection group ( $\mathrm{N}=8$ ), which received a full transection at $\mathrm{T} 7$ in the second operation.

In the second part of the study, we investigated the innervation of vertebrae downstream of the lesion site to determine if regenerated axons target single or multiple vertebrae. These experiments comprised 3 main groups, each of which were divided into 5 smaller sub-groups defined according to the site of the second operation: T8-T12. The first group was the operation group ( $\mathrm{N}=37$, Fig. 2A1-A2), in which all rats underwent 2 hemisection operations. The first operation was at $\mathrm{T} 7$ on the left side; 8 weeks later the second operation was carried out ipsilaterally at a single vertebra from T8-T12 depending on sub-group. The second group was the control group ( $\mathrm{N}=37$, Fig. 2B1-B5). All received a hemisection at $\mathrm{T} 7$ on the left side, and 8 
111 weeks later underwent a sham operation at a single vertebra at T8-T12 depending on sub-group.

112 The third was the sham operation group ( $\mathrm{N}=35$, Fig. 2C1-C5), in which rats in all sub-groups

113 received a sham operation at both time points.

\subsection{Hemisection operation}

\subsubsection{The first hemisection operation}

117 We generated the first hemisection operation according to previously published methods 118 (Arvanian et al. 2009). In the operation, we used sharp scalpel not scissors to separate along the 119 midline of spinal cord, which might cause less injury to the spinal cord. Before normal bladder control returned, we manually expressed the bladder of each rat once per day.

121

122

\subsubsection{The second hemisection operation}

The second hemisection operation was performed 8 weeks after the first hemisection operation. Except for the vertebra receiving a laminectomy, other operation procedures were the same as in the first hemisection operation.

\subsection{Basso, Beattie, Bresnahan (BBB) score tests in both hindlimbs}

Motor performance was scored were performed according to the well-known Open Field BBB locomotor scale (Basso et al. 1995). It was given each week after each hemisection operation. All of the BBB score tests, each lasting 5 minutes, were performed in an open field (diameter 150 $\mathrm{cm})$ with a wood floor. When we monitored the movement of the hindlimbs, all rats moved freely without any disturbance. The paw placement, joint movements, weight bearing, and coordination among the limbs were used to evaluate the BBB locomotion scale.

\subsection{Electrophysiological examinations :}


134 Before beginning electrophysiological examinations each rat was anesthetized. An 135 electrophysiological examination was given before and after each hemisection operation.

136

137

138

139

140

141

142

143

144

145

146

147

148

149

150

151

152

153

154

155

156

\subsubsection{Motor-evoked potential (MEP) studies on the body surface}

MEP examination and analyses were performed mainly according to previously published methods (Shen et al. 2016; Yin et al. 2015; Ziegler et al. 2011). Before the examination, the rat was in a relaxed state. Intramuscular electrode needles were implanted in the anterior tibial muscle (TA) and little toe abductor muscle (LTA) on both sides. There were 5 wires used for MEP examination, and each wire was connected to a stainless steel pin. The rostral-caudal locations of the wires were as follows: the first needle was in the midline $5 \mathrm{~mm}$ from the nose; the second needle was located subcutaneously in the midline of the head; the third needle was located subcutaneously in the mid-belly; the fourth needle was located in the middle of the muscle being tests; and the fifth needle was located in the tail $3 \mathrm{~cm}$ from the root. Stimulations of $10 \mathrm{~mA}$ at $1 \mathrm{~Hz}$ were administered once per time point: for $1 \mathrm{~ms}$ per stimulus. Each muscle received 3 standard stimuli, and the interval time was $30 \mathrm{~s}$.

\subsubsection{MEP on the spinal cord}

The purpose of the test in spinal cord was to observe the change of conduction from T7-T8 after the operation. Eelectrophysiological examinations involved stimulating microelectrodes and recording microelectrodes (Fig. 3F) (Arvanian et al. 2009; Schnell et al. 2011). The responses evoked by stimulating the ventral horn from the rostral end of $\mathrm{T} 7$ to the caudal end of $\mathrm{T} 8$ on the ipsilateral side were recorded on the same side or on the contralateral side. The stimulation electrode was positioned approximately $0.7 \mathrm{~mm}$ from midline, with a depth of $1.3 \mathrm{~mm}$, and an angle of $25-30^{\circ}$ from the vertical sagittal plane. The recording electrodes were positioned approximately $0.7 \mathrm{~mm}$ from middle line, with a depth of $1.3 \mathrm{~mm}$ and an angle of $15-20^{\circ}$ from the 
157 vertical sagittal plane. We used the average of two recordings for each side. There was a $30 \mathrm{~s}$

158 interval between the two stimuli. The ventral horn stimulus had a duration of $0.01 \mathrm{~ms}$ and a 159 current of $0.5 \mathrm{~mA}$ and was delivered at $1 \mathrm{~Hz}$.

160

161

\subsection{Criteria for excluding animals}

162 Rats were excluded from the research according to the following criteria: (1) death during or 163 after the operation; (2) signs of autophagia and/or a serious skin infection; (3) an edematous 164 hindlimb that would affect the BBB score test; (4) death during the electrophysiological 165 recordings.

\subsection{Statistics}

168

The statistical analysis was performed using SPSS database (version 19.0; SPSS Inc., Chicago, IL, USA). The BBB scores and electrophysiological examination data are shown as means \pm SEM. When the data agreed with the Bartley Ball Test, the repeated measures general linear model test was used to determine the overall differences in the different test times after the operation (1 week to 6,8 , or 16 weeks), followed by LSD (least significant difference) tests to make comparisons among groups. $\mathrm{P}$ values less than 0.05 were considered statistically significant.

\section{Results}

3.1 Part 1: Determination of an ipsilateral versus contralateral course

\subsubsection{BBB score $s$ after the first hemisection operation at $\mathrm{T} 7$}

After the first operation, none of the rats could move their left hindlimb or perform weight- 
180 bearing movements, while the right hindlimb could move slightly. The most significant and rapid 181 improvements in BBB scores of both hindlimbs occurred over the first 2 weeks. BBB scores

182 183

184

continued to increase through the $3 \mathrm{rd}$ week after the operation and then reached a plateau phase in the 4th week that persisted through the end of the evaluation period at week 8 (Fig. 4A).

\subsubsection{BBB scores after a second hemisection operation at $\mathrm{T} 7$}

A second hemisection at $\mathrm{T} 7$ had little effect on movement of either hindlimb in the ipsilateral group. In 7 of 8 rats, BBB scores recovered to the pre-second operation level on the 3rd day and the BBB scores of all rats recovered to the pre-second operation level by the end of the first week following the second operation (Fig. 4D). No significant differences in the BBB scores between the ipsilateral group and the control group were observed after the operation $(\mathrm{p}>0.05$, Fig. $4 \mathrm{C}$ and D).

After the second hemisection at T7 on the contralateral side, movement in both hindlimbs was instantly obstructed and then started to recover 2 weeks later. By the 4 th week, recovery entered a plateau phase. Compared to the rats in transection group, there were no significant differences in BBB scores of the ipsilateral group after the operation ( $p>0.05$, Fig. 4E and F). However, there were significant differences in BBB scores between the ipsilateral group and the contralateral group $(\mathrm{p}<0.05$, Fig. 4D and $\mathrm{E})$.

\subsubsection{Electrophysiological examinations in part 1 of the study}

After the second hemisection at T7, the latency periods in both TA muscles and both LTA muscles between rats in the ipsilateral group and control group were not significantly different ( $>0.05$, Fig. 5A-D). However, they were longer in the contralateral group than in the control group $(\mathrm{p}<0.05)$. There were no significant differences between the contralateral group and the transection group ( $\mathrm{p}>0.05)$. All experimental groups had significantly longer latency periods than 
203 the sham group $(\mathrm{p}<0.05)$.

204 There were not significant differences in latency periods in the spinal cord between the 205 sham, control, and ipsilateral groups ( $\mathrm{p}>0.05$, Fig. 6A and B, and Fig. 3A-C). However, the 206 latency period and wave amplitude disappeared in the contralateral and transection groups after 207 the second hemisection ( $\mathrm{p}>0.05$, Fig. 6A and B, and Fig. 3D1, D2, E1, E2).

3.2 Part 2: Determination of the involvement of vertebrae T8-T12

\subsubsection{BBB scores after a second hemisection operation at T8-T12}

In the T8 second hemisection group, BBB scores for the left hindlimb decreased slightly, while

BBB scores of the right hindlimb were barely affected. Approximately 3 weeks later, BBB scores of both hindlimbs recovered to the level before the operation ( $p>0.05$, Fig. 7A1). In comparisons of BBB scores 6 weeks after the second hemisection, the left hindlimbs in T8-T12 sub-groups displayed poorer and poorer improvement $(\mathrm{p}<0.05$, Fig. 7A1-A5). Seven of 8 rats in T12 operation sub-groups exhibited no left hindlimb movement 6 weeks after the operation. B1-B5).

BBB scores of both hindlimbs in all T8-T11 sub-groups in the sham operation group recovered completely by the second week after the second hemisection ( $p>0.05$, Fig. 7 C1-C4).

222 Compared to the T12 sub-group, the T8-T11 sub-groups displayed better improvement ( $<<0.05$, 223 Fig. 7 C1-C5).

\subsubsection{Electrophysiological examinations in part 2 of the study}

After the second hemisection in the operation group, the latency period in left TA muscles and 
226 left LTA muscles became progressively longer from the T8 sub-group to T12 sub-group $(\mathrm{p}<0.05$,

227 Fig. 8A, C).

228

2294 Discussion

230

231

232

233

234

235

236

237

238

239

240

241

242

243

244

245

246

247 248

\subsection{Effects of a second hemisection operation at $\mathrm{T} 7$}

Many previous animal experiments have shown that after a hemisection operation of the spinal cord, the transplantation of stem cells or various engineered tissue materials in the injury region can improve movement of both hindlimbs. Immunohistochemical examinations have also shown that the nerve fibers in regions rostral to the injury site increased and deeply innervated the lesion site. This indicates that nerves fibers penetrating into the area of injury probably play important roles in recovery after spinal cord injury (Estrada et al. 2014; Fan et al. 2010; Jee et al. 2012; McCall et al. 2012; Wright et al. 2011).

However, some studies using animal models have reached conflicting conclusions. An important example is the work of Courtine et al (Courtine et al. 2008), in which they carried out two successive lesions of the rat spinal cord and showed that the contralateral but not ipsilateral side was essential for recovery.

However, Courtine et al did not performed a second hemisection at the injury region, so whether nerve regeneration within the injury region was attributable to hindlimb movements recovery could not be ruled out (Courtine et al. 2008). Accordingly, in this study, we added an additional experimental group that received a second hemisection in the region of injury.

BBB scores for both hindlimbs decreased significantly after the first hemisection at $\mathrm{T} 7$, and 4 weeks later, the improvement reached a plateau. When the second hemisection operation was performed on the injury region, it had almost no effect on movement of either hindlimb $(\mathrm{p}>0.05$, 
249 Fig. 4C and D, Fig. 3B1, B2, C1, C2). The MEP results from body surface and spinal cord also

250 displayed no significant differences ( $\mathrm{p}>0.05$, Fig. 5A-D, Fig. 6A and B, Fig. 3C1, C2, D1, D2).

251 However, in the contralateral and transection groups, hindlimb movement on both sides

252 disappeared after the second operation and there were no significant difference between groups

253 at 8 weeks post-injury ( $p>0.05$, Fig. 4E and F). Moreover, MEP in the spinal cord disappeared

254 after the second operation in both groups (Fig. 6A, B and Fig. 3D1, D2, E1, E2). By contrast, we

255 found that, after the spinal cord transection operation MEP activities were still observed at the

256 body surface. For this reason, MEP examination of the spinal cord is a more accurate indicator of

257 injury and recovery than MEP at the body surface.

258 We thus conclude that the nerve repair in the injury region has no effect after the 259 hemisection operation at $\mathrm{T} 7$ on the ipsilateral side, and reparative responses involved nerve 260 fibers on the contralateral side.

261

262

263

\subsection{Effects of a second hemisection operation at T8-T12 on the ipsilateral side}

\subsubsection{T8 subgroup}

264 In T8 subgroup in operation group, the BBB scores of the left hindlimb decreased slightly, from the third week on, all rats returned to the level exhibited before the second operation (Fig. 7A1). Compared to the control group, there were significant differences in the BBB score of both hindlimbs $(\mathrm{p}<0.05$, Fig. 7A1, B1). And in the sham group, from the second week on, the sham operation almost had no effect to the movements of both hindlimbs. These results showed that the thoracic vertebra below and next to the spinal cord injury likely had a little assist in the recovery process, which may be affected by neuronal apoptosis around the injury site.

\section{$271 \quad$ 4.2.2 T9 subgroup}


272 In T9 subgroup in operation group, the BBB scores of both hindlimbs decreased slightly and

273 were approximately 1-2 points less than the level before the second hemisection in the $6^{\text {th }}$ week

274 (Fig. 7A2). But the reduction of BBB scores was a little more than in the sham group ( $\mathrm{p}<0.05$,

275 Fig. 7A2, C2) and were less than in the control group $(\mathrm{p}<0.05$, Fig. 7A2, B2). These results

276 showed that the spinal cord at thoracic vertebra T9 likely assisted with the recovery process to

277 some extent.

\section{$278 \quad$ 4.2.3 T10 subgroup}

279 In T10 subgroup in operation group, the BBB scores of left hindlimbs disappeared instantly and 280 began at the $1^{\text {st }}$ week (Fig. 7A3). When compared to the T9 subgroup within the operation group, 281 in the $6^{\text {th }}$ week, the left hindlimb recovered were worse (Fig. 7A2, A3). This finding showed that 282 the spinal cord at thoracic vertebra T10 played an important role in the spontaneous recovery of 283 the spinal cord injury, and the function could not be completely compensated for by other 284 segments of the spinal cord in 6 weeks.

\section{$285 \quad$ 4.2.4 T11 subgroup}

In T11 subgroup in operation group, the BBB scores of left hindlimb disappeared instantly and began at the $2^{\text {ed }}$ week (Fig. 7A4). When compared to the T10 subgroup within the operation group, the left hindlimb recovered were worse and the difference was significantly in 6 weeks (Fig. 7A3, A4). This finding showed that the spinal cord at thoracic vertebra T11 played a very important role in the spontaneous recovery of the spinal cord injury.

\subsubsection{T12 subgroup}

292

In T12 subgroup in operation group, the BBB scores of left hindlimb disappeared instantly and were approximately $0-1$ points from the $2^{\text {ed }}$ to $6^{\text {th }}$ week (Fig. 7A5). These results showed that the spinal cord at thoracic vertebra T12 played a major role in the spontaneous recovery of the spinal 
295

296

297

298

299

300

301

302

303

304

305

306

307

308

309

310

311

312

313

314

315

316

317

cord injury.

In summary, results of hemisection at a single vertebra from T8-T12 after an initial hemisection at T7 impaired hindlimb movement recovery in all instances, with the most pronounced effects occurring at T11-T12. Therefore, we conclude based on the data in part 2 of this study that, when caudal to the injury region area, spinal cord segments underlying the T8T12 vertebrae on the ipsilateral side all participated in the spontaneous recovery process after a hemisection operation at T7. In this case, the T12 vertebral area appeared to be the most important for nerve repair.

\subsection{Repair processes occurring rostral to the lesion}

Many findings have shown that, although the direct conduction pathway was destroyed in rats with a spinal cord injury, commands from brain conducted by the corticospinal tract (CST) can still be transmitted to the lumbar spinal cord below the lesion on the ipsilateral side (Bareyre et al. 2004; Courtine et al. 2008; Jankowska \& Edgley 2006; Kerschensteiner et al. 2004; van den Brand et al. 2012). After injury, an important mechanism of the spontaneous recovery process is thus that the structure and course of nerve fibers in the CST are remodeled such that they make contact with propriospinal neurons that form detour pathways bypassing the lesion (Nishimura \& Isa 2012; Pierrot-Deseilligny 2002; Rosenzweig et al. 2010; Zaaimi et al. 2012). However, the CST is not the only descending tract that affects movement and is not be the only projection system that conveys functional recovery (Han et al. 2013; Hurd et al. 2013). Spared reticulospinal fibers play an important role in the recovery process through spontaneous compensatory sprouting and increases in density after injury; they may also operate caudal to the lesion by enhancing indirect access to reticulospinal commands (Ballermann \& Fouad 2006; 
318 Zorner et al. 2014). Therefore, we hypothesize that the nerve fiber circuit underlying repair in 319 this study was composed of CST and reticulospinal fibers and propriospinal neurons. We 320 speculate that around the T12 vertebra, which was the most important for nerve repair, there

321 were more nerve fibers relative to the other vertebrae that crossed the midline from the 322 contralateral side to the injury side. However, more research, particularly nerve fiber tracing 323 experiments, is needed to confirm this.

\subsection{Repair processes occurring caudal to the lesion}

326

327

329

330

331

332

Tillakaratne et al. showed that rats exhibited spontaneous recovery via a step-wise process after a complete transection of the mid-thoracic spinal cord, even though the region caudal to the spinal cord lesion did not make any connections to the brain and in absence of descending tracts passing through the lesion (Tillakaratne et al. 2010). Thus, roles of activity of a locally acting central pattern generator (CPG), which is present in many species, are important to consider (Deliagina et al. 1999; Ekeberg \& Pearson 2005; Grillner 1985). While, our research argues for the presence of significant spontaneous locomotor recovery resulting from new forms of dynamic control in the spinal CPG from newly generated or remodeled descending tracts, the local CPG still may play the primary role in recovery following spinal cord injury (Rossignol et al. 2007).

The CPG of the spinal cord is located in the lumbar enlargement at about the T10-12 segments (Magnuson et al. 1999). The second part of this study showed that areas closer to T12 vertebra are more important for nerve repair. Therefore, we could conceivably use various treatments to reinforce the role of $\mathrm{CPG}$ and the nerve fibers that connect to it to promote recovery. However, given that a second hemisection operation at T9 on the ipsilateral side could 
341 also impair hindlimb movements, which could not recover to pre-operation levels, the CPG is

342 likely not the only factor playing a role in the recovery process.

\subsection{Study Limitations}

345 These studies assessed spontaneous recovery from spinal cord injury, but not the impact of any 346 treatment. Further research is needed to confirm if similar results are observed in the case of 347 using a therapeutic intervention within the same experimental injury paradigm; potential 348 therapies include targeting neuroinflammation, transplantation of engineered tissue materials, 349 stem cells, neurotrophic factors, or genetic modifications. We also did not directly assess the 350 path of newly projected nerve fibers to more conclusively and precisely define their course.

\section{Conclusions}

Our study demonstrates an anatomical mechanism for spontaneous repair processes caudal to

354

355

356

357

358

359

360

361

362

363

364

365

366 spinal cord injury sites in which regenerative fibers cross to the contralateral side, course around the lesion, and then re-cross the midline innervating all caudal, ipsilateral vertebrae with T12 being most important. If we inject stem cells, neurotrophic factors, drugs in the spinal cord around the injury region more than only in the injury region, it might had more effect in the recovery process. Further studies should investigate therapeutic approaches that enhance this process and identify the molecular mechanisms that control it.

\footnotetext{
6 References

Arvanian VL, Schnell L, Lou L, Golshani R, Hunanyan A, Ghosh A, Pearse DD, Robinson JK, Schwab ME, Fawcett JW, and Mendell LM. 2009. Chronic spinal hemisection in rats induces a progressive decline in transmission in uninjured fibers to motoneurons. Exp Neurol 216:471-480. 10.1016/j.expneurol.2009.01.004
} 
367

368

369

370

371

372

373

374

375

376

377

378

379

380

381

382

383

384

385

386

387

388

389

390

391

392

393

394

395

396

397

398

399

400

401

402

403

404

405

406

407

Ballermann M, and Fouad K. 2006. Spontaneous locomotor recovery in spinal cord injured rats is accompanied by anatomical plasticity of reticulospinal fibers. Eur J Neurosci 23:1988-1996. 10.1111/j.14609568.2006.04726.x

Bareyre FM, Kerschensteiner M, Raineteau O, Mettenleiter TC, Weinmann O, and Schwab ME. 2004. The injured spinal cord spontaneously forms a new intraspinal circuit in adult rats. Nat Neurosci 7:269-277. 10.1038/nn1195

Basso DM, Beattie MS, and Bresnahan JC. 1995. A sensitive and reliable locomotor rating scale for open field testing in rats. Journal of neurotrauma 12:1-21.

Courtine G, Song B, Roy RR, Zhong H, Herrmann JE, Ao Y, Qi J, Edgerton VR, and Sofroniew MV. 2008. Recovery of supraspinal control of stepping via indirect propriospinal relay connections after spinal cord injury. Nat Med 14:69-74. 10.1038/nm1682

Deliagina T, Orlovsky G, Selverston A, and Arshavsky Y. 1999. Neuronal Mechanisms for the Control of Body Orientation inClione I. Spatial Zones of Activity of Different Neuron Groups. Journal of neurophysiology 82:687-699.

Ekeberg O, and Pearson K. 2005. Computer simulation of stepping in the hind legs of the cat: an examination of mechanisms regulating the stance-to-swing transition. I Neurophysiol 94:4256-4268. 10.1152/jn.00065.2005

Estrada V, Brazda N, Schmitz C, Heller S, Blazyca H, Martini R, and Muller HW. 2014. Long-lasting significant functional improvement in chronic severe spinal cord injury following scar resection and polyethylene glycol implantation. Neurobiol Dis 67:165-179. 10.1016/j.nbd.2014.03.018

Etlin A, Blivis D, Ben-Zwi M, and Lev-Tov A. 2010. Long and short multifunicular projections of sacral neurons are activated by sensory input to produce locomotor activity in the absence of supraspinal control. J Neurosci 30:10324-10336. 10.1523/JNEUROSCI.1208-10.2010

Fan J, Xiao Z, Zhang H, Chen B, Tang G, Hou X, Ding W, Wang B, Zhang P, Dai J, and Xu R. 2010. Linear ordered collagen scaffolds loaded with collagen-binding neurotrophin-3 promote axonal regeneration and partial functional recovery after complete spinal cord transection. I Neurotrauma 27:1671-1683. 10.1089/neu.2010.1281

Grillner S. 1985. Neurobiological bases of rhythmic motor acts in vertebrates. Science 228:143-149.

Han Q, Feng J, Qu Y, Ding Y, Wang M, So KF, Wu W, and Zhou L. 2013. Spinal cord maturation and locomotion in mice with an isolated cortex. Neuroscience 253:235-244. 10.1016/j.neuroscience.2013.08.057

Hurd C, Weishaupt N, and Fouad K. 2013. Anatomical correlates of recovery in single pellet reaching in spinal cord injured rats. Exp Neurol 247:605-614. 10.1016/j.expneurol.2013.02.013

Jankowska E, and Edgley SA. 2006. How can corticospinal tract neurons contribute to ipsilateral movements? A question with implications for recovery of motor functions. The Neuroscientist 12:67-79.

Jee MK, Jung JS, Choi JI, Jang JA, Kang KS, Im YB, and Kang SK. 2012. MicroRNA 486 is a potentially novel target for the treatment of spinal cord injury. Brain 135:1237-1252. 10.1093/brain/aws047

Kerschensteiner M, Bareyre FM, Buddeberg BS, Merkler D, Stadelmann C, Bruck W, Misgeld T, and Schwab ME. 2004. Remodeling of axonal connections contributes to recovery in an animal model of multiple sclerosis. J Exp Med 200:1027-1038. 10.1084/jem.20040452

Magnuson DS, Trinder TC, Zhang YP, Burke D, Morassutti DJ, and Shields CB. 1999. Comparing deficits following excitotoxic and contusion injuries in the thoracic and lumbar spinal cord of the adult rat. Experimental 
neurology 156:191-204.

McCall J, Weidner N, and Blesch A. 2012. Neurotrophic factors in combinatorial approaches for spinal cord regeneration. Cell Tissue Res 349:27-37. 10.1007/s00441-012-1388-6

Nishimura $Y$, and Isa T. 2012. Cortical and subcortical compensatory mechanisms after spinal cord injury in monkeys. Exp Neurol 235:152-161. 10.1016/j.expneurol.2011.08.013

Pierrot-Deseilligny E. 2002. Propriospinal transmission of part of the corticospinal excitation in humans. Muscle Nerve 26:155-172. 10.1002/mus.1240

Reed WR, Shum-Siu A, and Magnuson DS. 2008. Reticulospinal pathways in the ventrolateral funiculus with terminations in the cervical and lumbar enlargements of the adult rat spinal cord. Neuroscience 151:505517. 10.1016/j.neuroscience.2007.10.025

Rosenzweig ES, Courtine G, Jindrich DL, Brock JH, Ferguson AR, Strand SC, Nout YS, Roy RR, Miller DM, Beattie MS, Havton LA, Bresnahan JC, Edgerton VR, and Tuszynski MH. 2010. Extensive spontaneous plasticity of corticospinal projections after primate spinal cord injury. Nat Neurosci 13:1505-1510. 10.1038/nn.2691

Rossignol S, Schwab M, Schwartz M, and Fehlings MG. 2007. Spinal cord injury: time to move? J Neurosci 27:1178211792. 10.1523/JNEUROSCI.3444-07.2007

Schnell L, Hunanyan AS, Bowers WJ, Horner PJ, Federoff HJ, Gullo M, Schwab ME, Mendell LM, and Arvanian VL. 2011. Combined delivery of Nogo-A antibody, neurotrophin-3 and the NMDA-NR2d subunit establishes a functional 'detour' in the hemisected spinal cord. Eur J Neurosci 34:1256-1267. 10.1111/j.14609568.2011.07862.x

Shen Y, Cao X, Shan C, Dai W, and Yuan TF. 2016. Heroin Addiction Impairs Human Cortical Plasticity. Biol Psychiatry. 10.1016/j.biopsych.2016.06.013

Shi Z, Ren H, Luo C, Yao X, Li P, He C, Kang JX, Wan JB, Yuan TF, and Su H. 2015. Enriched Endogenous Omega-3 Polyunsaturated Fatty Acids Protect Cortical Neurons from Experimental Ischemic Injury. Mol Neurobiol. 10.1007/s12035-015-9554-y

Tan Y, Ren H, Shi Z, Yao X, He C, Kang JX, Wan JB, Li P, Yuan TF, and Su H. 2016. Endogenous Docosahexaenoic Acid (DHA) Prevents Abeta1-42 Oligomer-Induced Neuronal Injury. Mol Neurobiol 53:3146-3153. 10.1007/s12035-015-9224-0

Tillakaratne NJ, Guu JJ, de Leon RD, Bigbee AJ, London NJ, Zhong H, Ziegler MD, Joynes RL, Roy RR, and Edgerton VR. 2010. Functional recovery of stepping in rats after a complete neonatal spinal cord transection is not due to regrowth across the lesion site. Neuroscience 166:23-33. 10.1016/j.neuroscience.2009.12.010

van den Brand R, Heutschi J, Barraud Q, DiGiovanna J, Bartholdi K, Huerlimann M, Friedli L, Vollenweider I, Moraud EM, Duis S, Dominici N, Micera S, Musienko P, and Courtine G. 2012. Restoring voluntary control of locomotion after paralyzing spinal cord injury. Science 336:1182-1185. 10.1126/science.1217416

Wright KT, El Masri W, Osman A, Chowdhury J, and Johnson WE. 2011. Concise review: Bone marrow for the treatment of spinal cord injury: mechanisms and clinical applications. Stem Cells 29:169-178. 10.1002/stem.570

Yin Z, Shen Y, Reinhardt JD, Chen CF, Jiang X, Dai W, Zhang W, Machado S, Arias-Carrion O, Yuan TF, and Shan C. 2015. $5 \mathrm{~Hz}$ Repetitive Transcranial Magnetic Stimulation with Maximum Voluntary Muscle Contraction Facilitates Cerebral Cortex Excitability of Normal Subjects. CNS Neurol Disord Drug Targets 14:1298-1303.

Zaaimi B, Edgley SA, Soteropoulos DS, and Baker SN. 2012. Changes in descending motor pathway connectivity after corticospinal tract lesion in macaque monkey. Brain 135:2277-2289. 10.1093/brain/aws115 
449 Ziegler MD, Hsu D, Takeoka A, Zhong H, Ramon-Cueto A, Phelps PE, Roy RR, and Edgerton VR. 2011. Further

450

451

452

453

454

455 evidence of olfactory ensheathing glia facilitating axonal regeneration after a complete spinal cord transection. Exp Neurol 229:109-119. 10.1016/j.expneurol.2011.01.007

Zorner B, Bachmann LC, Filli L, Kapitza S, Gullo M, Bolliger M, Starkey ML, Rothlisberger M, Gonzenbach RR, and Schwab ME. 2014. Chasing central nervous system plasticity: the brainstem's contribution to locomotor recovery in rats with spinal cord injury. Brain 137:1716-1732. 10.1093/brain/awu078 
456

457

458
459

460

461

463

464

465 462 The sham group. (B) The control group. (C) The ipsilateral group. (D) The contralateral group.

\section{Figure Legends}

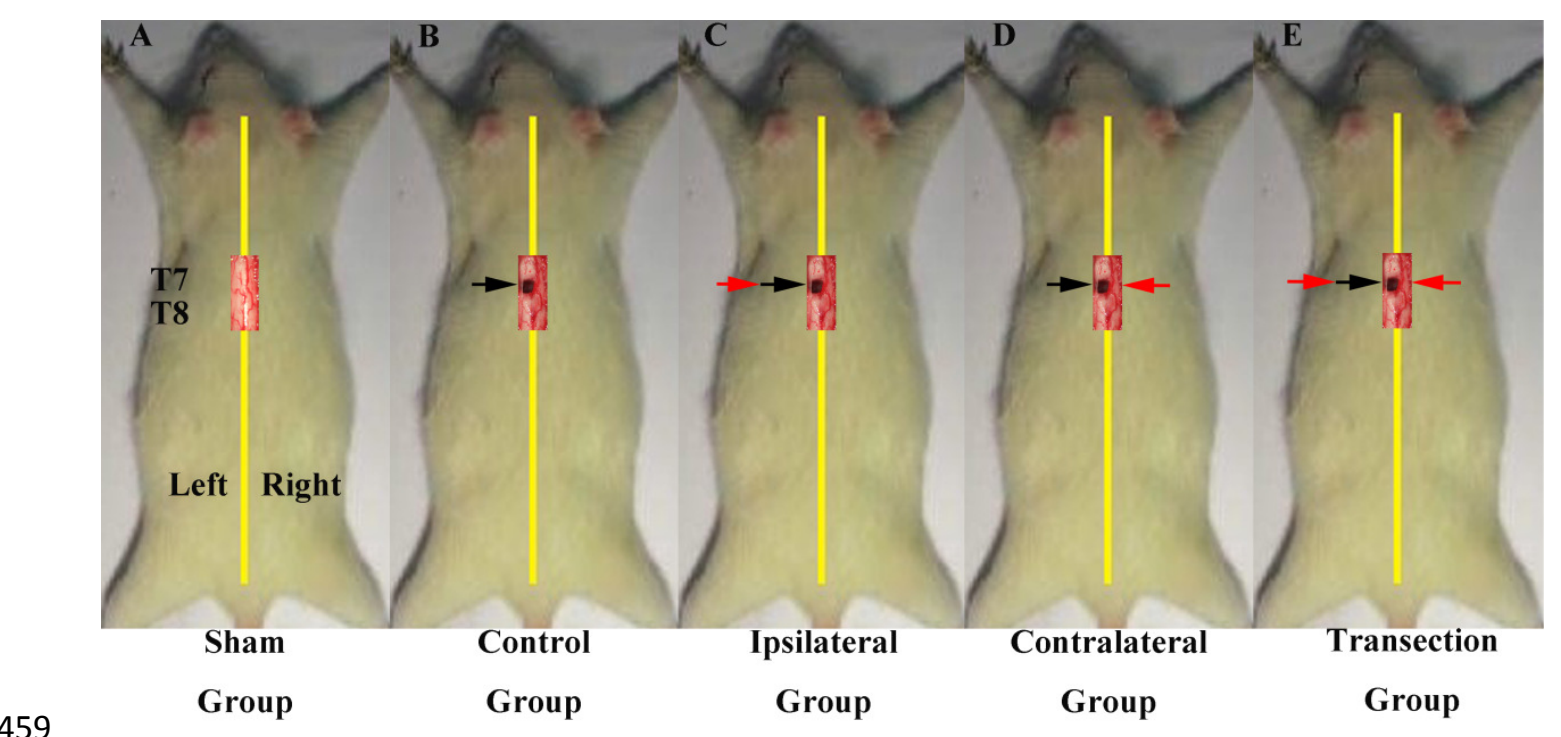

Fig 1. Operations in first part of this study. All the operations were made at T7. A black arrow 61 and a red arrow indicates the level of the first and second hemisection operation separately. (A) (E) The transection group. 

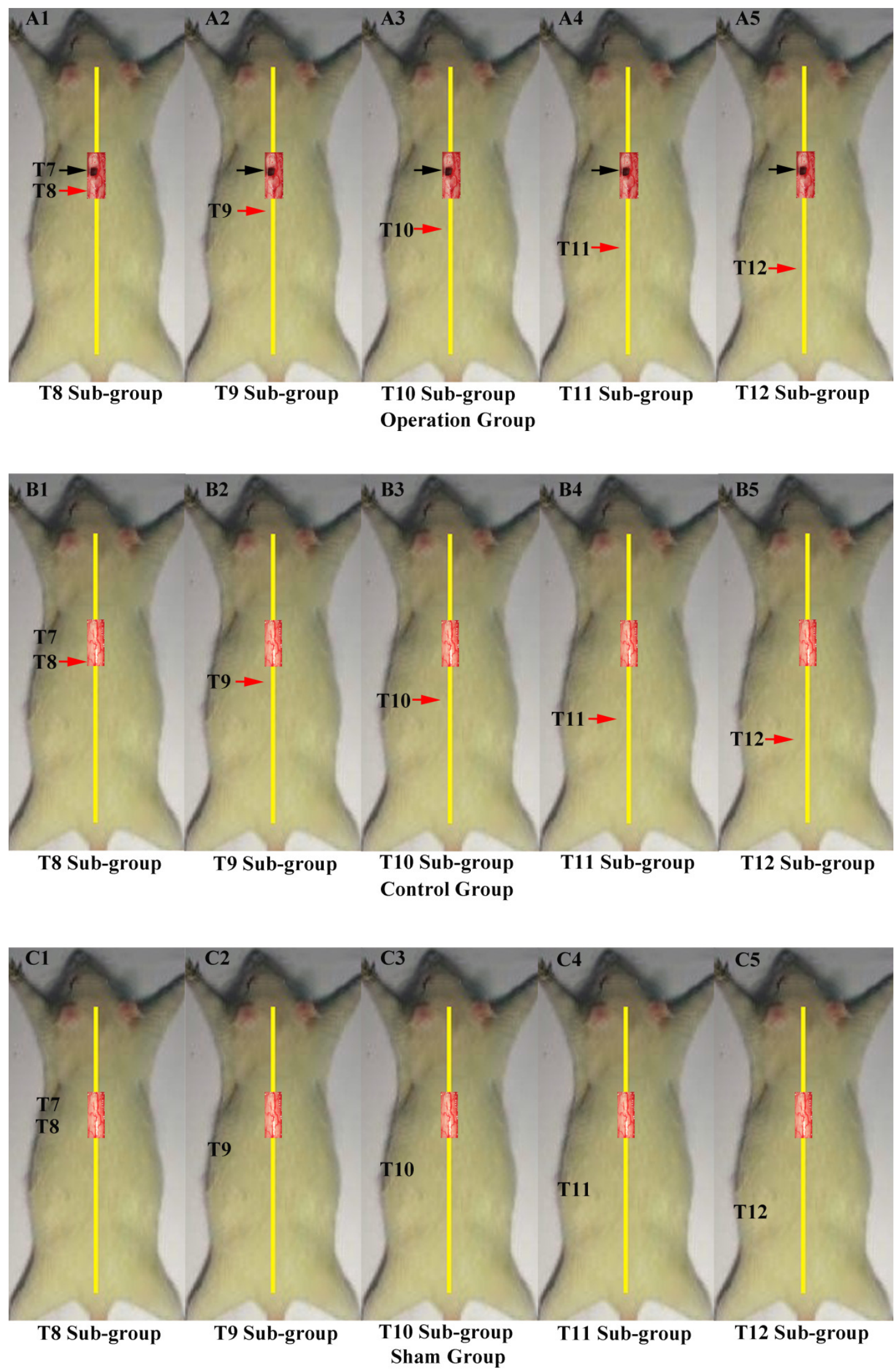
467 Fig 2. Operations in second part of this study. A black arrow indicates the level of the first 468 hemisection operation; a red arrow indicates the level of the second hemisection operation. (A1469 A5) T8-T12 sub-groups in the operation group. (B1-B5) T8-T12 sub-groups in the control group. 470 (C1-C5) T8-T12 sub-groups in the sham group. 


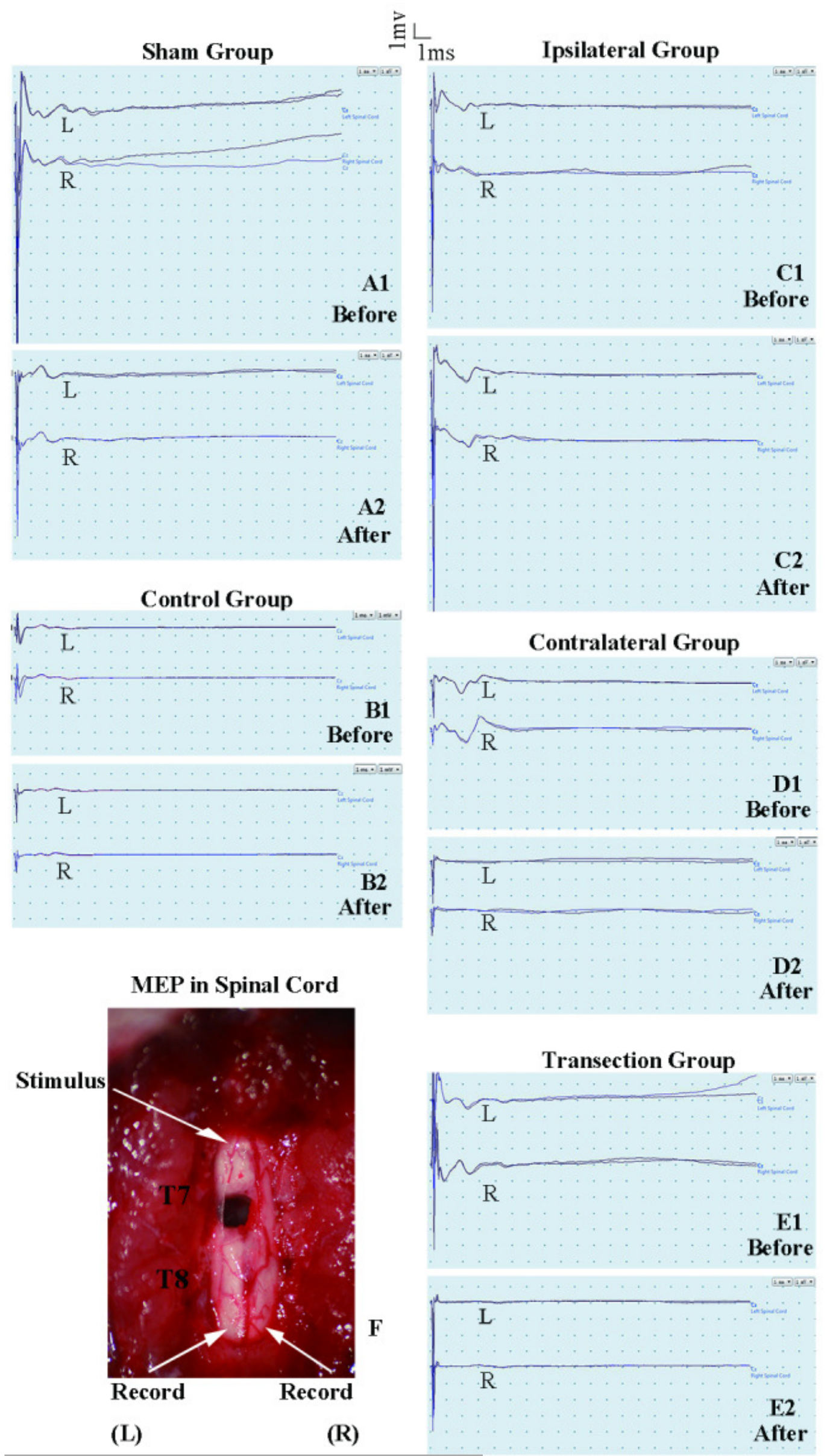


473 Fig 3. Electrophysiological examinations of the spinal cord in first part of this study before and 474 after the second hemisection operation at T7: (A) Sham group, (B) Control group, (C) Ipsilateral 475 group, (D) Contralateral group, (E) Transection group.

476

477

478 

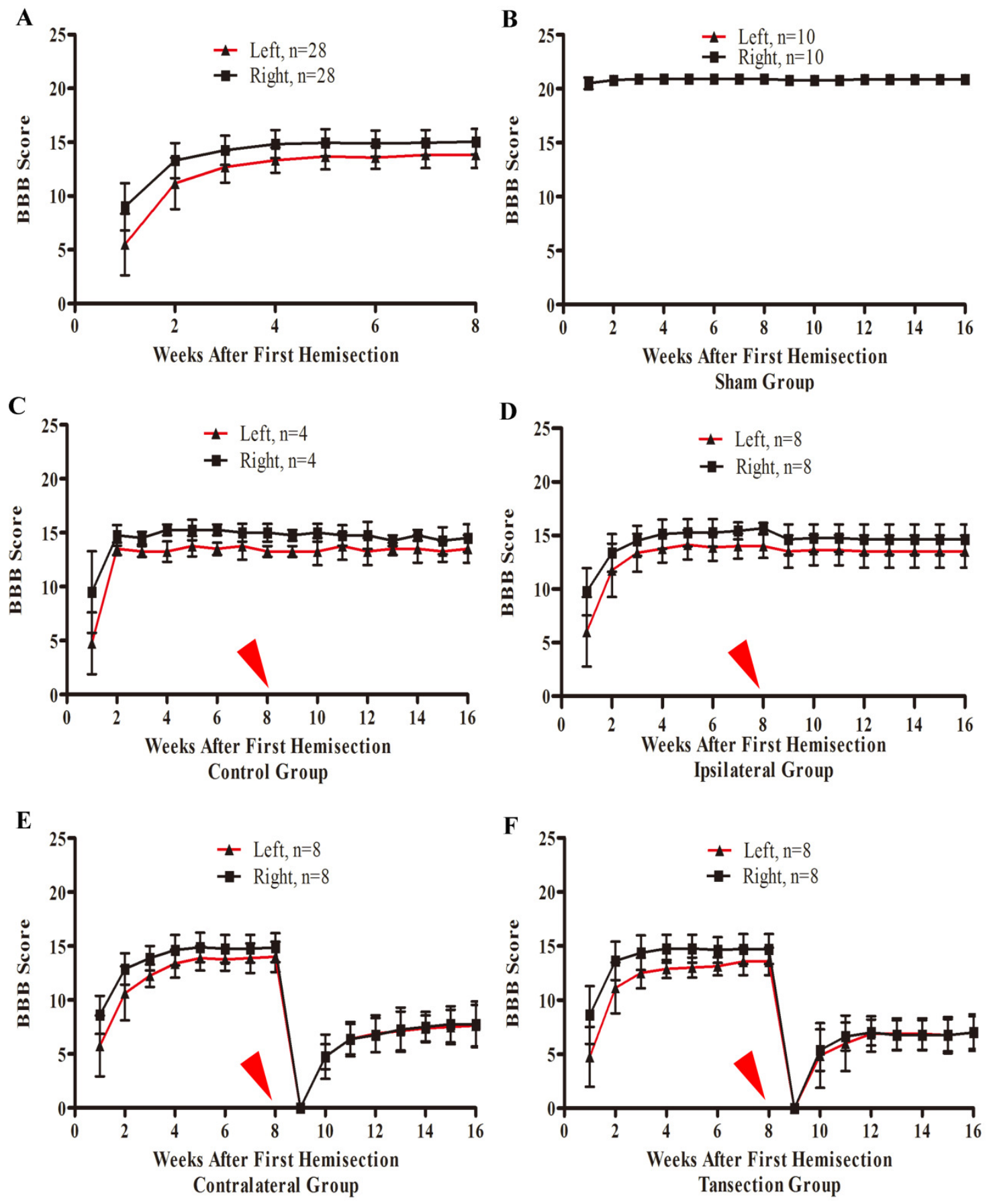

480 Fig 4. The BBB scores for part 1 of the study. A red arrow indicates the time point for the second

481 hemisection. (A) The trend in BBB scores of all rats before the second hemisection operation.

482 (B-E) Trends in BBB scores:(B) Sham group, (C) Control group, (D) Ipsilateral group, (E)

483 Contralateral group, (F) Transection group. Data are presented as mean $\pm \mathrm{SEM}$. 
484

485
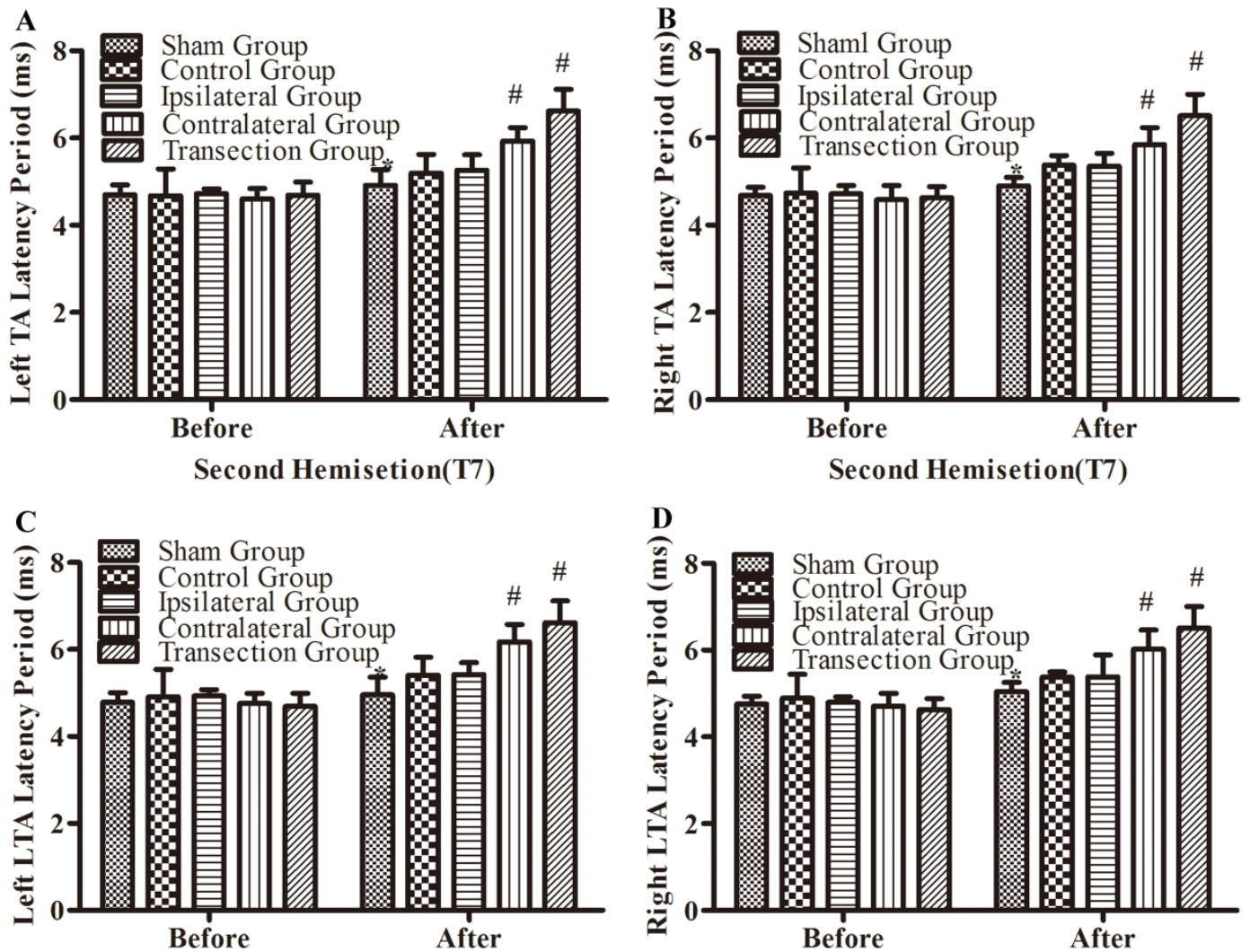

486

Second Hemisetion(T7)

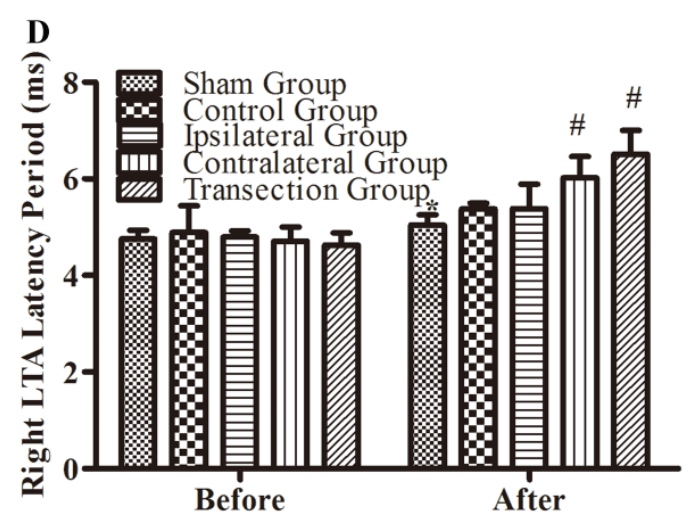

Second Hemisetion(T7)

487

Fig 5. Electrophysiological examinations (MEP, latency periods) in body surface in part 1 part of 488 this study. (A-D) The latency periods in both TA muscles and both LTA muscles. *, $\mathrm{P}<0.05$, 489 compared to the control group. \#, $\mathrm{P}<0.05$, compared to the other 4 groups. Data are presented as 490 mean \pm SEM.

491

492

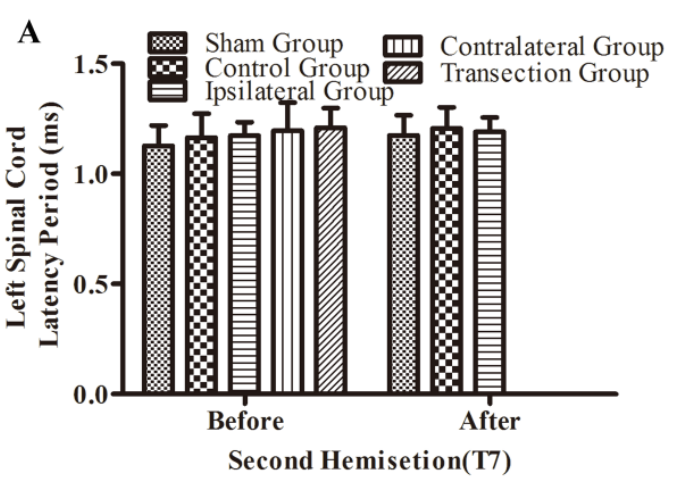

Second Hemisetion(T7)

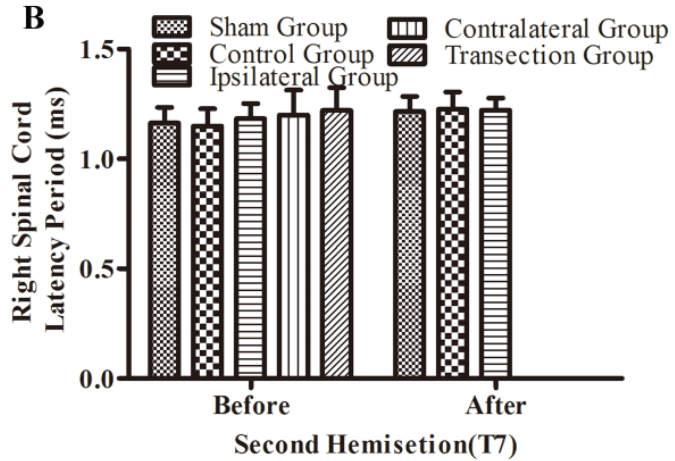

Fig 6. 
494 Electrophysiological examinations (MEP, latency periods) of the spinal cord in part 1 of this 495 study. (A-B) The latency periods on both sides of the spinal cord before and after the second 496 hemisection operation. Data are presented as mean \pm SEM.

497

498
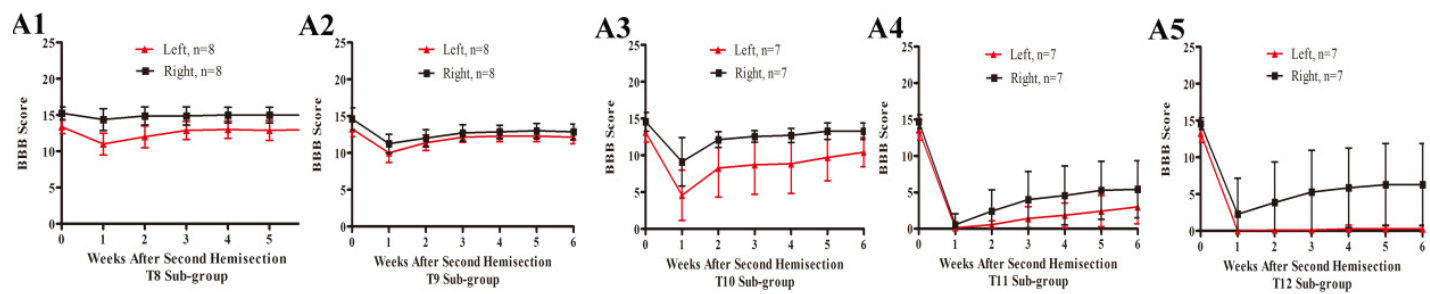

Operation Group
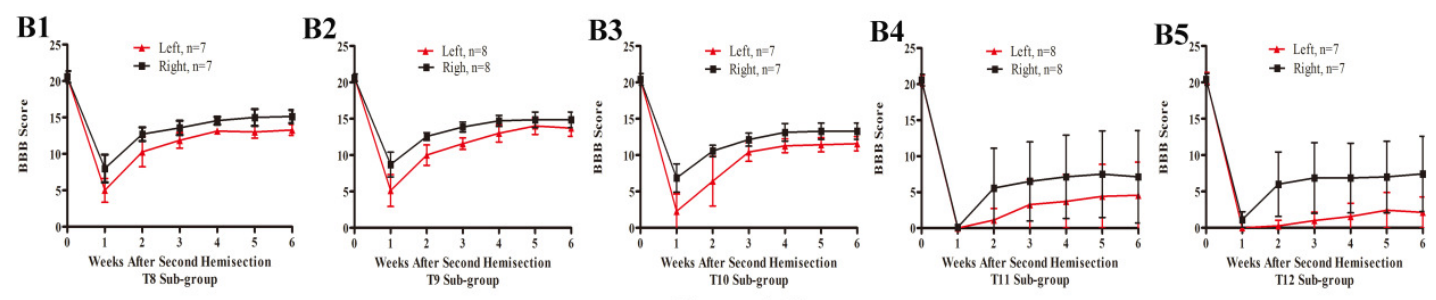

Control Group

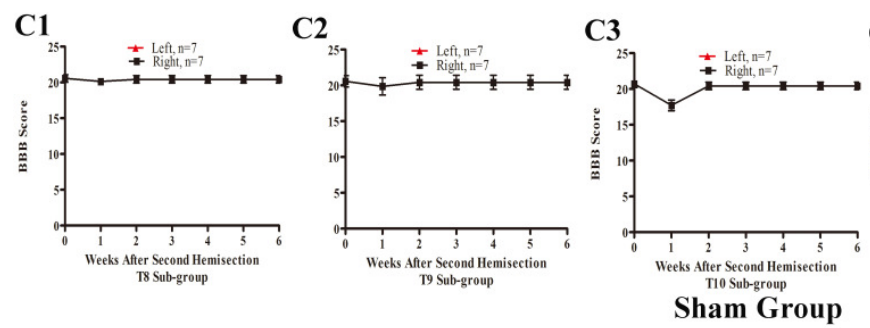

499

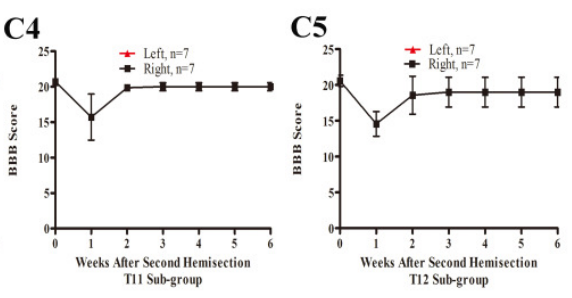

500

Trends in BBB scores tendency for the T8 to T12 sub-groups in part 2 of the study: (A1-A5)

501

Operation group, (B1-B5) Control group, (C1-C5) Sham group. Data are presented as mean \pm

502 SEM. 

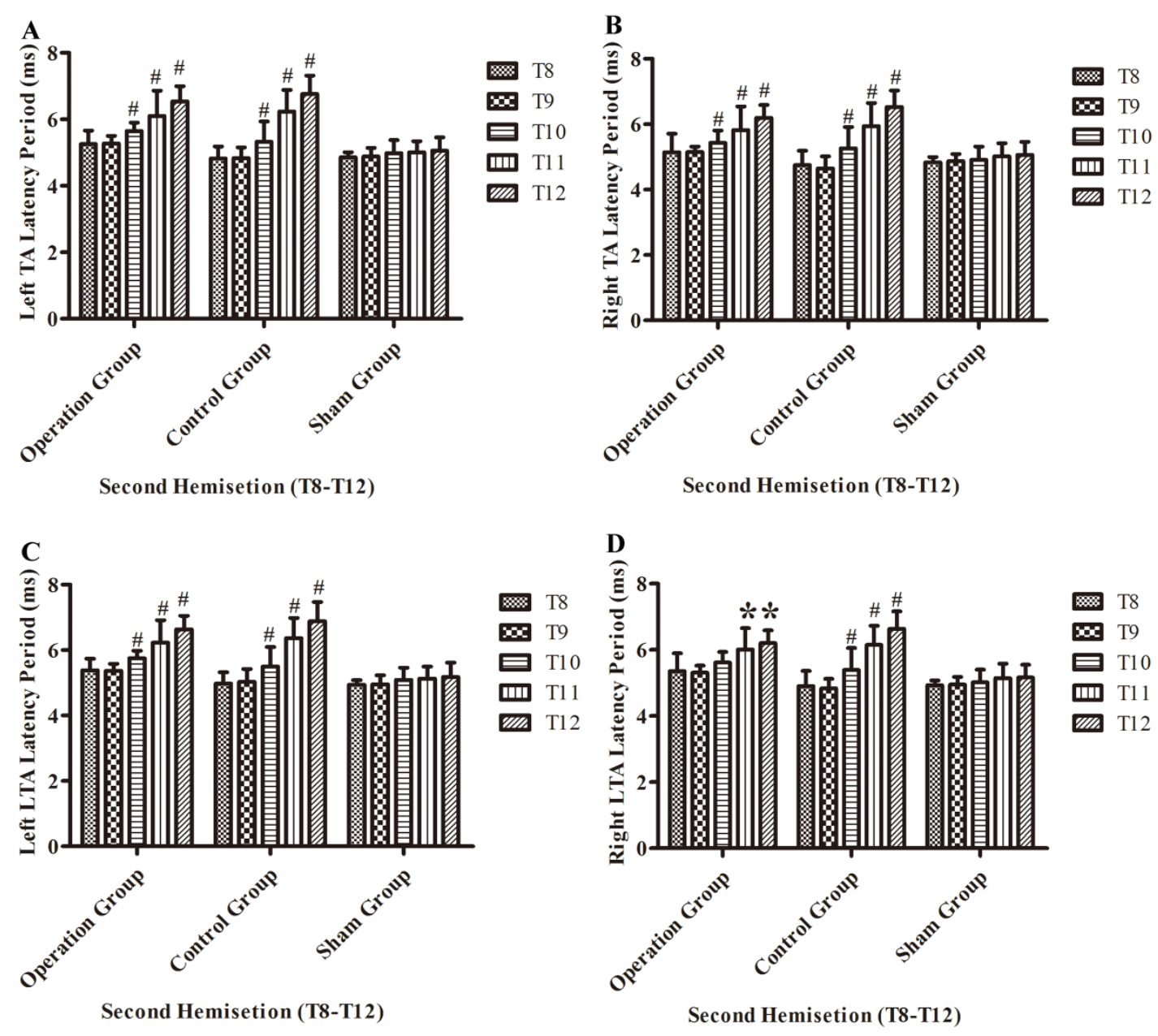

504

505

Fig 8. Electrophysiological examinations (MEP, latency periods) in part 2 of this study after the

506 second hemisection operation at the (A) left TA muscle, (B) right TA muscle, (C) left LTA

507 muscle, (D) right LTA muscle. *, $\mathrm{P}<0.05$, compared to T8 sub-group in each main group. \#,

$508 \mathrm{P}<0.05$, compared to the other 4 sub-groups. Data are presented as mean $\pm \mathrm{SEM}$. 